\title{
Efficacy of Liposomal Cytarabine in the Treatment of Leptomeningeal Metastasis of Breast Cancer
}

\author{
Elena Laakmann Isabell Witzel Volkmar Müller \\ Department of Gynecology, University Medical Center Hamburg-Eppendorf, Hamburg, Germany
}

Established Facts

- About 5\% of patients with metastatic breast cancer develop leptomeningeal metastasis (LM).

- The prognosis of patients with LM and breast cancer is poor with a reported median overall survival 3.3-4.5 months.

- Intrathecal therapy is one of the treatment options of the LM.

\section{Novel Insights}

- The case describes an extremely long progression-free survival (18 months+) of a patient with LM of breast cancer treated with intrathecal liposomal cytarabine.

- The represented cases suggest a rapid neurological response by a timely treatment start for the LM.

\section{Keywords}

Breast cancer - Leptomeningeal metastasis .

Liposomal cytarabine

\section{Summary}

Background: Breast cancer is one of the most frequent causes of leptomeningeal metastasis (LM) among solid tumors in adults. The prognosis of patients with LM is poor. A treatment option is the intrathecal administration of liposomal cytarabine. Case Reports: The first case represents a 53-year-old woman with LM of breast cancer. A neurological response was achieved shortly after the start of the multimodal treatment including intrathecal liposomal cytarabine. The response duration reached 79 days. The second case represents a 48-year-old woman with LM of breast cancer treated with intrathecal liposomal cytarabine as a part of a multimodal treatment approach. A neurological response was achieved in the first 2 weeks of treatment and is still lasting after
18 months. Conclusion: These cases suggest that a rapid response to a prompt start of treatment with intrathecal liposomal cytarabine in patients with LM of breast cancer is feasible. In 1 case an extremely long progressionfree survival (18+ months) was achieved. The cases support the efficacy of liposomal cytarabine in the management of LM in breast cancer patients as a part of a multimodal treatment approach and underline the need for further investigations in this specific cohort.

(c) 2017 S. Karger GmbH, Freiburg

\section{Introduction}

Leptomeningeal metastasis (LM) is defined by tumor cell spread to the leptomeninges and subarachnoid space [1]. Breast cancer, lung cancer, and melanoma are the most frequent causes of LM

\section{KARGER}

(c) 2017 S. Karger GmbH, Freiburg 
among solid tumors in adults [2]. About $5 \%$ of patients with metastatic breast cancer develop LM [1]. In particular, patients with a triple-negative and lobular primary breast cancer have a propensity to develop metastases in the leptomeninges [3]. The prognosis of patients with LM and breast cancer is poor: medial survival times of between 3.3 and 4.5 months have been reported [3-5].

The local evidence-based treatment options for LM of breast cancer are the intrathecal therapy with either liposomal cytarabine, methotrexate or thiotepa, and radiotherapy of the brain [6]. Liposomal cytarabine (Depocyte ${ }^{\circledR}$ ) is a frequently used compound for intrathecal administration. The mechanism of action is based on the inhibition of the DNA synthesis [1]. The administration interval of 2 weeks, which is less frequent compared to the other compounds, is beneficial for the patients $[1,7]$. The long half-life of liposomal cytarabine provides a therapeutic concentration in the intracerebral fluid of up to 12 days [2].

Until now, the efficacy of liposomal cytarabine has mostly been proved in cohorts of patients with LM of mixed primary solid tumors or lymphoma. The response rates of $26-72 \%$ and median time to neurological progression of 58-105.5 days were observed in these cohorts [8-10]. The efficacy of the liposomal cytarabine in breast cancer patients with LM has been analyzed in a smaller number of trials: the median time to neurological progression of 49 days and 2.1 months was shown by Jaeckle et al. [7] and Le Rhun et al. [11].

Here we present 2 case reports concerning the efficacy of liposomal cytarabine in patients with a leptomeningeal disease and breast cancer.

\section{Case Reports}

\section{Case 1}

In January 2016, a 53-year-old woman with a metastatic breast cancer developed vomiting, dizziness and unilateral facial hypoesthesia. The general condition of the patient was reduced with an ECOC performance status of 1. At the time the patient was treated with endocrine therapy with fulvestrant and denosumab for bone metastases of hormone receptor-positive HER2-negative invasive lobular breast cancer. The magnetic resonance imaging (MRI) of the brain showed signs of LM, the diagnosis was confirmed by analysis of the cerebrospi nal fluid (12/3 malignant cells in the initial lumbar puncture).

Intrathecal therapy with liposomal cytarabine $(50 \mathrm{mg}$, intrathecal, every 2 weeks), a concomitant systemic therapy with capecitabine and cranial radiotherapy were started shortly after the diagnosis of LM. The symptoms of the patient improved already 2 weeks after therapy start. The number of malignant cells in the cerebrospinal fluid after the third intrathecal drug administration reduced to $4 / 3$. In the further course of treatment, however, a symptom recurrence was observed after 73 days. 5 intrathecal injections were given. No clinically significant side effects were observed under the treatment with liposomal cytarabine. The complaints of the patient during the therapy (e.g. nausea) were probably associated with the leptomeningeal disease. 79 days after the diagnosis of LM the patient died.

\section{Case 2}

In December 2014, a 48-year-old woman with a metastatic hormone receptor-positive HER2-negative invasive lobular breast cancer (to the bones) presented with dizziness, nausea, vomiting, impaired vision and weight loss. The general condition of the patient was reduced with an ECOC of 2. The ongoing therapy was tamoxifen, goserelin and denosumab. The MRI of the brain showed arachnoid enrichment compatible with leptomeningeal disease as well as unilateral orbital metastasis. The analysis of the cerebrospinal fluid confirmed the diagnosis of the LM (27/3 malignant cells in the initial lumbar puncture).

Systemic therapy with paclitaxel, orbital radiotherapy and intrathecal therapy with liposomal cytarabine (50 mg, intrathecal, every 2 weeks) was started within 10 days after the diagnosis of LM. The patient's symptoms decreased noticeably within the first 2 weeks of treatment. The side effects during the treatment were headache, nausea, dizziness, double images. The therapy was, however, in total well tolerated.

Due to the stable general condition the application of the liposomal cytarabine was paused after the sixth intrathecal drug administration, a re-induction was appointed in case of worsening of the symptoms. The systemic application of paclitaxel continued. The cerebral MRIs at all control time points $(6 / 2015$, $9 / 2015,1 / 2016,6 / 2016$ ) showed no signs of the leptomeningeal disease; the orbital metastasis was stable in size.

Up to now, after 18 months of therapy, the patient remains on treatment with paclitaxel, has a stable extracranial disease and no signs of CNS progression assessed per imaging or clinically.

\section{Discussion}

Our case reports support the evidence of the efficacy of the liposomal cytarabine in the management of breast cancer patients with a leptomeningeal disease: in 1 case a survival rate comparable to the published data was achieved; in the other, a remarkably long survival was achieved. In both cases a comparatively quick response concerning the neurological symptoms was seen. In our opinion this might be also explained by a rapid treatment start after diagnosis of LM. The duration of response, however, was different in the presented cases.

In the case with a long progression-free survival, an initially combined treatment approach, i.e. systemic treatment, intrathecal drug administration and radiotherapy, might have a positive influence on the outcome of the patient. A positive influence of an initially combined treatment was confirmed in the investigation of Le Rhun et al. [11] and is supported by the literature review of Scott et al. [12]. Some of the current known negative predicting factors could not be confirmed in this case.

The clinical cases reported here and the research background underline the importance of a multimodal individualized approach in the treatment of leptomeningeal disease in breast cancer, and emphasizes the clinical need concerning further research in this field.

The efficacy of the intrathecal treatment with liposomal cytarabine should be further evaluated in large homogeneous breast cancer patient cohorts as the biological behavior of breast cancer differs from other primary tumors. Factors predicting response to the intrathecal therapy and survival should be evaluated further, as up to now controversial views exists on this issue.

Further investigations should also be performed to identify the appropriate role of concomitant radiotherapy of the brain in addition to intrathecal treatment, as well as duration of treatment after clearance of malignant cells. As prospective trials are difficult to perform, registries should help to increase the knowledge about LM. Therefore, an online registry has been set up for LM [13]. 


\section{Disclosure Statement}

The authors declare no conflict of interest. Expense allowance was covered by POMME-med GmbH.

\section{References}

1 Mack F, Baumert BG, Schafer N, et al.: Therapy of leptomeningeal metastasis in solid tumors. Cancer Treat Rev 2016;43:83-91.

2 Le Rhun E, Taillibert S, Chamberlain MC: Carcinomatous meningitis: Leptomeningeal metastases in solid tumors. Surg Neurol Int 2013;4:S265-288.

3 Niwinska A, Rudnicka H, Murawska M: Breast cancer leptomeningeal metastasis: Propensity of breast cancer subtypes for leptomeninges and the analysis of factors influencing survival. Med Oncol 2013;30:408.

4 de Azevedo CR, Cruz MR, Chinen LT, et al.: Meningeal carcinomatosis in breast cancer: Prognostic fac tors and outcome. J Neurooncol 2011;104:565-572.

5 Lee S, Ahn HK, Park YH, et al.: Leptomeningeal metastases from breast cancer: Intrinsic subtypes may affect unique clinical manifestations. Breast Cancer Res Treat 2011;129:809-817.
6 Thill M, Liedtke C, on behalf of the AGO Breast Committee: AGO recommendations for the diagnosis and treatment of patients with advanced and metastatic breast cancer: update 2016. Breast Care 2016;11:216222.

7 Jaeckle KA, Phuphanich S, Bent MJ, et al.: Intrathecal treatment of neoplastic meningitis due to breast cancer with a slow-release formulation of cytarabine. $\mathrm{Br} \mathrm{J}$ Cancer 2001;84:157-163.

8 Garcia-Marco JA, Panizo C, Garcia ES, et al.: Efficacy and safety of liposomal cytarabine in lymphoma patients with central nervous system involvement from lymphoma. Cancer 2009;115:1892-1898.
9 Glantz MJ, Jaeckle KA, Chamberlain MC, et al.: A randomized controlled trial comparing intrathecal sustained-release cytarabine (DepoCyt) to intrathecal methotrexate in patients with neoplastic meningitis from solid tumors. Clin Cancer Res 1999;5:3394-3402.

10 Summary of product characteristics. EMA.

11 Le Rhun E, Taillibert S, Zairi F, et al.: A retrospective case series of 103 consecutive patients with leptomeningeal metastasis and breast cancer. J Neurooncol 2013;113:83-92

12 Scott BJ, Kesari S: Leptomeningeal metastases in breast cancer. Am J Cancer Res 2013;3:117-126.

13 http://site.meningeosis.net/. 